\title{
RAZIONALITÀ POLITICA, VIRTÙ CIVICA E IDENTITÀ NAZIONALE
}

\author{
di Gian Enrico Rusconi
}

Il problema in termini generali e in due casi nazionali

La democrazia ideale è quella che combina funzionalità istituzionale, efficienza economico-amministrativa con il consenso e il lealismo dei cittadini. Quando un sistema democratico realizza queste condizioni ottimali, può dirsi un modello di razionalità politica. Naturalmente le democrazie realmente esistenti sono lontane da questo modello. Molte sono inefficienti nei loro apparati istituzionali, hanno cattive prestazioni amministrative, con pesanti conseguenze economiche e sociali e contraccolpi negativi in termini di consenso. Ė il caso della democrazia italiana da anni viziata da inefficienze e disfunzionalità che, aggravate da sistematici meccanismi di corruzione, hanno dato luogo a fenomeni di disaffezione civico-politica senza precedenti.

$\mathrm{Ma}$ anche in un sistema politico-istituzionale, ben altrimenti solido come quello della Germania, sono venute alla luce inattese inefficienze connesse ai problemi della riunificazione, con ricadute negative sullo «spirito pubblico» (risorgenza della xenofobia e del razzismo) ed effetti economico-finanziari a livello europeo e internazionale.

Abbiamo così davanti due esempi di deficit di razionalità politica, anche se di natura diversa: di carattere istituzionale e strutturale in Italia, di tipo prevalentemente gestionale in Germania. In entrambi i casi però la qualità delle disfunzioni mette in luce carenze di autorevolezza/autorità dei governi che riaprono problemi di identificazione e integrazione sociale e civica ad un livello così profondo da porre in discussione il senso e il valore dell'appartenenza nazionale. Questo fatto ha colto di sorpresa politologi, sociologi e storici che avevano considerato esaurita $e$ irrilevante qualunque funzione integrativa e identitaria della nazione storica oggi.

RIVISTA ITALIANA DI SCIENZA POLITICA / a. XXIV, n. 1, aprile 1994 
Naturalmente le fenomenologie del caso tedesco e del caso italiano sono diverse. In Germania l'appello al solidarismo nazionale e civico, la tematica della coscienza nazionale e del patriottismo sono espliciti e centrali nel dibattito pubblico. Si invoca il senso di appartenenza storica nazionale tra i cittadini dell'ovest e dell'est per sostenere o surrogare i limiti della politica governativa. Questa operazione non manca di sollevare vivaci contestazioni, mentre sullo sfondo la risorgente xenofobia, per quanto circoscritta, non esita a esibire simboli e parole «nazionali».

In Italia il panorama è di segno opposto e contrastato. Qui il postulato dell'integrazione solidale della nazione storica viene contestato da un movimento politico, il leghismo, che vede il superamento dell'attuale crisi politica, economica e morale nell'abbandono o nella radicale revisione della forma statuale unitaria della nazione. La rivendicazione leghista di un «federalismo macroregionale» non si presenta semplicemente come una proposta di funzionalità/razionalità politica, bensì come un'operazione che con maggiore o minore reticenza mira alla svalorizzazione dell'identità storica nazionale. Anche se su questo punto la confusione è grande, non si può negare che tra le spinte culturali del leghismo c'è la voglia o la invenzione di identità collettive e forme di integrazione neo-territoriale regionale in alternativa a quella nazionale. L'ipotesi secessionistica si configura quindi come una minaccia credibile, a dispetto se non addirittura a motivo di una dinamica comunicativa basata su dichiarazioni avventurose e mirate alla provocazione o smentite controproducenti, che innescano controaccuse strumentali in un gioco verbale dalle conseguenze imprevedibili. La questione della ristrutturazione regionale-federale sta per diventare decisiva in Italia, anche perché polemicamente caricata da aspettative senza precedenti.

Di fronte a questa situazione gran parte delle forze politiche tradizionali reagisce in modo fermo ma sostanzialmente rituale in difesa dell'unità nazionale. In compenso l'appello ai valori della nazione viene enfaticamente ripreso ed esibito dal movimento di destra in sensibile crescita.

Non esaminerò in questa sede i tratti e i moventi socio-culturali del successo della destra in Italia, effimero o duraturo che sia. Mi limito a segnalare alcune evidenze. Innanzitutto la ripresa del motivo «nazionale» non offre argomenti originali o innovatori capaci di contrastare il leghismo; anzi si presta alla facile 
accusa di essere una copertura del vecchio statalismo centralista e assistenzialista. Questa evidenza viene rafforzata dalla netta delimitazione geografica (centro e meridione) del successo della destra. Il risultato è che sotto il segno della questione nazionale si accentua la virtuale spaccatura del paese.

Non intendo qui sviluppare analisi dirette sostantive di questa problematica. Ritengo invece utile riflettere sistematicamente sui concetti che possono costituire l'impalcatura teorica di un'analisi empirica dei fenomeni menzionati: razionalità politica, civismo e identità nazionale.

\section{Quesiti e assunti di partenza}

Cominciamo dal quesito se e come alla soluzione dei problemi di efficienza politica di una democrazia, segnalati sopra, possa contribuire l'attivazione della virtù civica. E quindi se l'esercizio del civismo possa considerarsi espressione di razionalità politica. Per affrontare questo punto occorre partire dall'assunto che esistano (o possano esistere) «virtù civiche», cioè comportamenti di lealtà politica esercitati dai cittadini in circostanze normali e in condizioni di difficoltà particolari che richiedono «costi» speciali in nome della comune appartenenza ad una comunità politica. Dal momento che «civismo» e «solidarismo civico» sono termini inflazionati nella retorica etico-politica, è bene precisare che con essi qui non vengono intesi comportamenti «altruistici» motivati da sentimenti religiosi o morali genericamente umanitari. Non si tratta di negare l'esistenza o di sottovalutare l'enorme rilevanza collettiva di queste motivazioni di altruismo. Ma il nostro intento è quello di identificare la specificità civico-politica del vincolo solidale tra i cittadini.

Ebbene, proprio in questa ottica, è importante precisare che nel cuore e nella testa della gente un comportamento civico così definito non discende direttamente o necessariamente dal principio universalistico della cittadinanza democratica, bensì presuppone il riferimento ad una qualche comunità storica di appartenenza.

A partire dal XIX secolo, con intensità crescente, questo referente comunitario politico è stato la «nazione», comunque definita e organizzata nella forma-Stato. Essa ha svolto (o ha preteso svolgere) la funzione principale di obbligazione civica e di 
identificazione collettiva. Soltanto dalla seconda metà del XX secolo lo ha fatto in modo sempre meno stringente, al punto che presso gli studiosi si è largamente diffusa l'opinione che in quasi tutte le democrazie cosiddette avanzate il senso d'identità nazionale sia in declino (un fatto considerato quasi da tutti come uno dei pochi fenomeni inequivocabilmente positivi del nostro tempo).

Da qui il secondo quesito. Se si sono gradualmente e irreversibilmente esauriti i sentimenti di identità nazionale, con cui è storicamente cresciuto il civismo tradizionale, oggi le virtù civiche vanno pensate e promosse a prescindere dal senso di una qualunque appartenenza territoriale?

Questa tesi è falsificata dalla insorgenza di rivendicazioni di cittadinanze subnazionali, regionali, locali che esigono in modo perentorio lealtà che riproducono su scala ridotta i tratti aggressivi ed etnocentrici del nazionalismo storico. Addirittura esse mettono in pericolo i valori civici democratici che sono stati salvaguardati, bene o male, dalle nazioni storiche. Come vedremo più avanti, alla nazione democratica si contrappongono etnodemocrazie regionali.

A questo punto dobbiamo chiederci se ci troviamo di fronte a fenomeni semplicemente regressivi oppure ad autentiche esigenze identitarie che invitano a riformulare i criteri attraverso cui si sono prodotte storicamente l'identificazione con la nazione, l'idea di cittadinanza e le virtù civiche ad esse connesse. Questa ottica rimette in discussione - almeno nel caso italiano - anche i fondamenti di legittimazione e di autorità dello Stato, quantomeno nella forma del governo sinora esistente.

Per affrontare questa problematica dobbiamo fare un passo indietro e prendere le mosse da alcune considerazioni, apparentemente molto astratte, sull'agire politico e sulla razionalità delle sue componenti utilitario-strumentali e identitarie, per poi ritornare sulle ragioni del civismo e del senso di appartenenza nazionale.

Il lettore verificherà quanto questo impianto analitico sviluppi argomentazioni e valutazioni che si discostano da quelle presenti nel dibattito tra «liberali» e «comunitaristi», nonostante qualche affinità con alcuni passaggi di quella discussione ${ }^{1}$.

${ }^{1}$ Cfr. l'antologia curata da Alessandro Ferrara (1992), in particolare i saggi di Alasdair MacIntyre e di Charles Taylor. A quest'ultimo saggio farò qualche accenno nella n. 20. 


\section{La razionalità strategica}

Classicamente, «razionale» si definisce il comportamento di un soggetto o di un attore che è strumentale al raggiungimento di un determinato obiettivo. Questo tipo di razionalità viene riconosciuto e imputato (dall'osservatore) ad attori che sono capaci di intenzionalità e di piani di azione «strategici». Questo primo significato di agire strategico è omologo a quello di agire strumentale rispetto ad uno scopo. Ma c'è una seconda accezione di agire strategico che si riferisce all'interazione o all'interdipendenza delle scelte di due o più attori che agiscono simultaneamente e si riconoscono reciprocamente la capacità di avere piani d'azione. Strategica è pertanto la razionalità di attori che hanno di mira un rapporto reciproco ottimale (o almeno soddisfacente), da cui dipende congiuntamente il loro benessere. La qualità dell'utile o lo stato di benessere raggiunto da un attore dipendono cioè dal tipo di relazione instaurata con l'altro.

Questo concetto collettivo di razionalità strategica si applica all'agire politico. La sua specificità tuttavia è data da quel particolare complesso di rapporti o interazioni che la filosofia e la scienza politica tradizionale qualificano con la fenomenologia del potere (ruoli di comando/obbedienza, di rappresentanza, di legittimazione ecc.). Politiche sono pertanto le interazioni e le interdipendenze collettive nelle quali la razionalità strategica si rivela nelle forme del potere (anche nella loro versione democratica) ${ }^{2}$.

Attori della politica, così intesa, possono essere soggetti diversi. Nella concezione democratica si prende avvio dall'individuo-cittadino che, dotato di diritti civili, sociali e politici, li usa attivamente nel ruolo di elettore e di partecipante alla vita politica. Ma attori politici sono anche i soggetti collettivi che agiscono unitariamente (partiti, gruppi di interesse) e gli apparati istituzionali (quali i governi o i parlamenti). Non va infine dimenticato che l'agire politico rimanda sempre alle coordinate

2 A questo livello di astrazione sono compatibili tutte le definizioni classiche del potere a cominciare da quella weberiana di «possibilità di trovare obbedienza, presso certe persone, ad un comando che abbia un determinato contenuto» - anche se tipicamente Weber si pone sempre nell'ottica di chi detiene effettivamente o virtualmente il potere. Credo che l'impostazione del problema della razionalità politica, formulata nel testo, sia compatibile anche con la definizione sartoriana della politica come «sfera delle decisioni "collettivizzate" sovrane, coercitivamente sanzionabili e senza uscita». Cfr. Sartori $(1990,216)$. 
del tempo (la storia e la sua memoria) e dello spazio (il territorio e i suoi confini).

Tutti gli attori politici ora menzionati praticano strategie nel doppio significato di piani di azione mirati al proprio scopo ma insieme calcolati sulle mosse, sulle aspettative e sulle presunte risorse degli altri attori ${ }^{3}$.

Il paradigma dell'agire strategico così inteso complica il modo convenzionale di intendere la relazione tra «mezzo» e «scopo». Abbiamo già detto che nelle interazioni strategiche il «mezzo» è più propriamente un piano o una sequenza di mosse programmate (con maggiore o minore rigidità). Ora aggiungiamo che lo «scopo» o l'obiettivo rispecchia più propriamente «l'ordine di preferenze» dell'attore, che risponde a determinati criteri di razionalità interna (transitività e connessione delle preferenze). Un tale ordine di preferenza non solo consente di determinare in modo più articolato e preciso «lo scopo» dell'attore, ma rimanda a quella che con un'espressione ingannevolmente semplice chiamiamo la «identità» degli attori e quindi ai suoi processi di formazione e di trasformazione. In questo modo l'identità e i suoi processi entrano a formare il comportamento strategico - nella sua accezione più complessa e completa. L'espressione «strategia identitaria» o «comunicativa» non è (o non dovrebbe essere) una metafora o una figura retorica, ma un modo cogente di connettere due dimensioni dell'agire.

\section{Strategia identitaria e comunicazione}

Introdotto via strategica il concetto di identità, non ho intenzione di addentrarmi nel labirinto delle sue definizioni psicologiche, culturali, sociologiche. Qui mi limito ad assumere

${ }^{3}$ Data la pluralità e la eterogeneità dei soggetti in gioco (singoli cittadini, partiti, istituzioni, gruppi di interesse o altro) le situazioni di interazione che si creano sono inevitabilmente complesse e conflittuali. Per coglierle e razionalizzarle è a disposizione (dell'attore e dell'osservatore) l'impianto concettuale dei «giochi strategici» - uno strumento che a torto le scienze sociali e politologiche nostrane continuano a guardare con toni di sufficienza. In questo quadro, particolarissima rilevanza hanno i problemi delle incongruenze e dei contrasti tra le scelte dettate dalle razionalità individuali $\mathrm{e} i$ risultati collettivi, considerati cioè dal punto di vista della razionalità complessiva di tutti gli attori in gioco. Questi problemi sono sistematicamente affrontati nelle teorie e nei modelli del «paradosso del voto». Cfr. i classici testi di K.J. Arrow, in particolare Equilibrio, incertezza e scelta sociale (1987). Per il lettore italiano non specialista segnalo i saggi di W.H. Riker e S.J. Brams in Rusconi $(1989,107-194)$. 
l'identità come una «riserva di senso» di cui dispone l'attore quando entra in interazione con altri attori. Questa riserva di senso, che è precondizione dell'agire dotato di senso, appunto, dell'attore, ha una sua autonomia e peculiarità.

Sarebbe tuttavia un errore se da queste caratteristiche deducessimo nel modo d'agire dell'attore una incompatibilità o noninterferenza tra risorse di senso dell'identità e logiche del comportamento strumentale. Il dispiegamento di senso dell'identità infatti si materializza nell'agire sempre come «l'ordine delle preferenze» che orienta l'attore nella interazione strategica con gli altri.

Non è vero dunque che le identità sono inattaccabili e inaccessibili a logiche strumentali. In realtà dalle proprie «riserve di senso» gli attori traggono risorse che, dispiegandosi strategicamente (nel significato illustrato sopra), retroagiscono sugli stessi processi identitari, dando luogo cioè a mutazioni e assestamenti dell'identità stessa.

Si può e si deve quindi parlare di «strategia identitaria» in senso proprio, quando il piano d'azione prevede o produce una risistemazione o un mutamento degli ordini di preferenza, con i quali un attore entra in rapporto con altri. In altre parole, l'attore cambia le proprie preferenze ovvero modifica, entro certi limiti, la propria identità per poter vincere o per non perdere, a seconda della posta in gioco.

In questa operazione compare il linguaggio. Si delinea così il rapporto tra strategia identitaria e strategia comunicativa, da considerare in tutte le sue forme soprattutto performative (minaccia, promessa, impegno ecc. $)^{4}$.

Nella definizione della strategia comunicativa o della comunicazione tout court va evitato un doppio unilateralismo. $\mathrm{Da}$ un lato infatti essa non può essere ridotta ad un mero set di informazioni, grazie al quale gli attori semplicemente danno inizio alla interazione e la conducono con margini più o meno ampi di incertezza e di calcolo razionale di probabilità. Un attento studio dell'interazione linguistica porta facilmente a mettere in luce le incongruenze e $\mathrm{i}$ limiti di una concezione meramente strumentale della razionalità ${ }^{5}$. Dall'altro lato, però, la comunicazione non può neppure essere collocata su un piano epistemologicamente privilegiato - il solo delegato all'acquisizione di

${ }^{4}$ Su questo tema punto di partenza obbligato rimane J.L. Austin (1962).

5 Cfr. Midgaard (1980). 
una intesa con la controparte (la Verständigung di Jürgen $\mathrm{Ha}$ bermas) presuntivamente sottratta alla logica stategico-strumentale e alle sue parzialità.

Vedremo più avanti come la strategia dell'intendersi, insieme con quella dell'argomentazione imparziale-universalistica, possa contribuire a spiegare il nesso tra razionalità politica e virtù civica nei termini della nostra problematica. Per il momento accontentiamoci di affermare che è scorretto o ingenuo assegnare alla logica comunicativa contenuti e valori di una intesa in politica che si traduce immediatamente in solidarismo civico; mentre alla logica strategico-strumentale sono imputati i soli obiettivi ed effetti dell'autoaffermazione egoistica, parziale, antisolidale.

Se consideriamo poi l'agire politico nelle coordinate del territorio e della storia, entro cui prende corpo la nazione, entriamo in un'altra dimensione. E vero che oggi le coordinate spazio-temporali di riferimento sembrano mutare per dimensioni e per profondità rispetto a quelle tracciate dalla nazione storica: si ridisegnano su organismi sopranazionali oppure su territorialità subnazionali. Ma questo, lungi dal rendere irrilevanti le componenti strumentale e identitaria dell'agire politico descritte sopra, rende ancora più impegnativo capire come queste componenti si esprimano nelle nuove forme neo-, sub- o anti-nazionali e come retroagiscano sui contenuti delle virtù civiche.

\section{Il caso tedesco: $i$ problemi dell'integrazione unitaria}

Prima di andare avanti nel nostro ragionamento, proviamo ad applicare quanto stiamo dicendo alla situazione tedesca. Più esattamente esaminiamo la dinamica interattiva in cui si trovano i cittadini tedeschi dell'est e dell'ovest dopo la riunificazione. Si tratta, beninteso, di uno schema molto semplice, che parte dal presupposto che l'obiettivo dei tedeschi orientali sia quello di tirarsi fuori dai disastri, ereditati dal socialismo, con strategie che utilizzano in modo ottimale le opportunità offerte dalla politica governativa per superare lo squilibrio esistente tra le due parti della Germania. Questa strategia, apparentemente tutta strumentale, possiede anche forti componenti identitarie, nel senso che la riconversione alla democrazia e al capitalismo dei tedeschi orientali non può avvenire senza una profonda trasformazione dei loro precedenti «ordini di preferenza», senza una 
alterazione di aspetti importanti della loro identità collettiva formatasi nella ex-DDR.

La dinamica instauratasi a partire dal $1989 / 91$ presenta due aspetti incrociati. I tedeschi orientali sentono di pagare costi soggettivi identitari altissimi in termini di complesso di inferiorità, di frustrazione di aspettative, di depressione. Specularmente, però, nella percezione di molti tedeschi occidentali i concittadini orientali godono di una posizione di fruitori «a buon mercato» (di free-rider) di opportunità materiali, «pagate» dai tedeschi occidentali. Questa situazione potrebbe essere formalizzata come un gioco nel quale il «bene comune» della riunificazione tedesca è raggiunto o, meglio, viene pagato con costi materiali e immateriali distribuiti in modo asimmetrico. Gli abitanti della ex-DDR spuntano certamente vantaggi materiali ma insieme vengono mortificati nella loro autostima, nelle loro attese quando addirittura non sono denegati della loro precedente identità storica degradata a vergogna. I tedeschi occidentali si sentono gravati di costi materiali, senza che la riunificazione appaia (almeno agli occhi di molti) un arricchimento della loro identità collettiva. Dal momento che ognuna delle strategie messe in atto dagli attori può considerarsi a suo modo razionale, ci troviamo dinanzi ad una collisione di razionalità ovvero ad un deficit di razionalità collettiva.

E corretto che questo difetto venga imputato primariamente alla politica - più esattamente a carenze di prestazione e di efficienza, non solo economica ma anche culturale, da parte del governo che non ha saputo attuare un «gioco cooperativo» percepito come vantaggioso da tutti i partecipanti. Un difetto di governo e di autorità politica in senso forte.

Proprio di fronte a queste difficoltà si è acceso in Germania il dibattito sulla necessità di un nuovo «patto solidale» tra i cittadini dell'ovest e dell'est, per il quale è stato usato (riabilitato) anche il concetto di «patriottismo» ${ }^{6}$. E naturalmente il governo ha tutto l'interesse a usare questa risorsa per sostenere e legittimare la propria linea politica.

6 Si veda in particolare la serie degli articoli apparsi sulla «Die Zeit» a partire dal gennaio 1993, aperta dall'intervento di Robert Leicht Obne Patriotismus geht es nicht. 


\section{Le ragioni del civismo e la sua strategia}

«Patriottismo» è una denominazione impegnativa, storicamente pregiudicata e quindi controversa per definire la virtù collettiva del solidarismo civico, invocata per scongiurare egoismi e particolarismi di parte. Si potrebbe parlare più sobriamente di superamento dei contrasti di razionalità individuali nella prospettiva di una razionalità collettiva.

Ma di nuovo: la virtù civica (patriottica) è un surrogato più o meno efficace o una componente a pieno titolo della razionalità collettiva democratica? In che senso, quindi, chi rifiuta il solidarismo civico può considerarsi non soltanto un cattivo cittadino ma anche un cattivo attore/comunicatore razionale?

Nella letteratura scientifica e filosofica ci sono vari modi di affrontare questi interrogativi. C'è un approccio utilitaristico che cataloga il solidarismo e in genere ogni virtù civica e collettiva appunto come una utilità sui generis. Questa cioè rientra nell'ordine di preferenza e di calcolo di individui perfettamente razionali. Questa problematica può essere messa a fuoco grazie agli strumenti della teoria dei giochi misti di cooperazione e conflitto, nei quali i benefici collettivi appaiono come payoff e utilità congiunte.

A questo approccio non interessa stabilire perché e quando ci sono individui dotati di ordini di preferenza che mettono al primo posto utilità o beni comuni, non fruibili cioè separatamente dal singolo, ma godibili congiuntamente. L'utilitarista si limita a prendere atto della frequenza di queste situazioni.

Chi ragiona in questo modo non è un cinico insensibile alla rilevanza sociale e politica delle virtù collettive. Semplicemente per lui queste virtù sono «razionali» soltanto in quanto si piegano e si spiegano in una logica utilitaria. Al di fuori di questa logica c'è il regno dell'irrazionalità, per quanto nobili e augurabili siano i suoi effetti.

Questa impostazione offre spiegazioni spesso convincenti. Infatti sovente il solidarismo civico risponde ad un calcolo sofisticato di utilità che vede nella mancata offerta della propria virtù la causa di un danno comune, che prima o poi coinvolge lo stesso calcolatore miope. Si tratta dunque di una strategia razionale nel senso che rinuncia ad un vantaggio individuale immediato (quale sarebbe l'astenersi dal pagare per il solidarismo) perché è incerto delle conseguenze negative indirette (o nel tempo) che ne deriverebbero; si accolla così un costo individua- 
le (sotto forma della propria «virtù») pur di evitare un costo collettivo (certo o molto probabile) peggiore per tutti ${ }^{7}$.

Una variante di questo schema presenta il solidarismo come una polizza di assicurazione basata non soltanto sul differimento della soddisfazione immediata, ma sull'aspettativa che anche altri attori si comportino nello stesso modo. Si instaura così una razionalità che è propria del comportamento cooperativo e che mette in moto una risorsa peculiare: la fiducia $^{8}$ reciproca tra gli attori e una comunicazione speciale tra essi.

Il limite di questo approccio utilitario alla cooperazione sta nel fatto che conta sul fattore speciale «fiducia», presupposto della cooperazione, senza preoccuparsi di come e quando esso possa essere prodotto. Non gli interessa come il fattore «fiducia» attinga a quella «riserva di senso», che costituisce l'identità stessa degli attori, e si disinteressa quindi anche delle strategie comunicative cui il fattore «fiducia» è strettamente legato. In altre parole, i modelli cooperativi sviluppati secondo la logica della rational choice seguono con perspicacia la dinamica della razionalità condizionale, che si basa sul calcolo delle probabilità dell'instaurarsi del coordinamento tra gli attori e dei suoi prerequisiti (compresa la fiducia). Ma ignorano le dinamiche soggettive, che ne sono alla base: queste sono lasciate o affidate ad altri approcci (alla «psicologia delle passioni»).

Ritengo invece che questo insieme di problemi, lungi dall'essere delegabile ad un campo di indagine separato, rientri a pieno titolo nello studio delle strategie identitarie e comunicative della politica.

Tra le risorse identitarie atte a creare le condizioni di fiducia reciproca, che sono alla base del comportamento cooperativo-solidale in una comunità nazionale, vanno annoverate quelle che attingono ad una comune memoria storica. C'è una controprova di questo fatto: i processi di desolidarizzazione collettiva o di disidentificazione nazionale cominciano dalla ritrattazione delle memorie storiche comuni, nazionali appunto.

Se alla luce di queste considerazioni ritorniamo alla questione del civismo/patriottismo, quale era suggerita dal caso tedesco, possiamo ora riformularla in termini più articolati. Quale peso hanno (o devono avere) nell'appello al civismo i motivi e

${ }^{8}$ Cfr. Riker (1980) e Gambetta (1989). 
gli incentivi utilitari? Quale peso hanno (o devono avere) le motivazioni «fiduciarie» che fanno riferimento a comuni matrici identitarie storico-culturali (dei tedeschi dell'ovest e dell'est), come presupposto per un comportamento reciprocamente cooperativo? Quale ruolo e posizione assumono invece le ragioni puramente universalistiche dedotte dal principio della cittadinanza democratica?

A questi interrogativi si può dare una risposta pragmatica, di buon senso, asserendo che l'efficacia e la razionalità della virtù civica dipendono dall'intreccio, di volta in volta variabile a seconda delle circostanze, delle tre dimensioni: utilitaria, storico-identitaria, universalistica.

Se vogliamo invece spostare il ragionamento ad un livello teorico più impegnativo, dobbiamo riconoscere che questo intreccio pragmatico di argomenti chiama in causa la sostanza della strategia comunicativa di cui si parlava sopra. Con essa il «comunicare» a favore del civismo (e più in generale per realizzare la razionalità politica) non può attenersi ai criteri del «discorso» habermasiano, presuntivamente costruito secondo regole immanenti all'intendersi linguistico (su questo tornerò criticamente nel prossimo paragrafo). La strategia comunicativa, così come emerge dalle riflessioni fatte sin qui, è la capacità di saper disporre nel discorso pubblico dell'intero quadro dei motivi di azione dei cittadini. Non si tratta di rinunciare al principio del primato razionale dell'argomento migliore, dell'argomentare imparziale e universalistico, ma di riconoscere che nella concretezza e contingenza dell'agire politico esso è costantemente intrecciato con altri motivi. In particolare sono frequenti gli «usi strategici dell'argomentare» che combinano ragionamenti imparziali e motivazioni parziali, senza l'esclusione di alcuni momenti manipolativi ${ }^{9}$.

E scorretto declassare tutto questo a patologia della comunicazione o a mera accidentalità che non va confusa con la natura tipico-ideale dell'agire comunicativo. Infatti è soltanto attraverso questi processi che si crea concretamente consenso e lealismo politico, e quindi si esercitano le virtù civiche.

9 Cfr. i lavori di Jon Elster in particolare The cement of society: a study of social order, (1989) e il recente, ora anche in italiano, Argomentare e negoziare, (1993a). Disgraziatamente nella traduzione di quest'ultimo gli importanti riferimenti a paragrafi e pagine del cit. The cement of society sono erroneamente e sistematicamente attribuiti ad un altro libro di Elster tradotto in italiano (Come si studia la società, 1993b) dove evidentemente il lettore non li trova. 


\section{Un excursus critico sulla Diskurstheorie di Habermas}

Uno degli interlocutori più autorevoli di questa problematica e della discussione sul patriottismo e la nazione tedesca è Jürgen Habermas ${ }^{10}$. Il filosofo/sociologo francofortese inquadra i suoi argomenti polemici contro quello che ritiene un pericoloso revival nazionalistico in Germania in una teoria generale dell'agire comunicativo o della Diskurstheorie. È opportuno pertanto fare qualche rilievo critico sulla trasposizione delle sue tesi filosofiche in una teoria della politica. Parlo delle tesi che fanno della «razionalità comunicativa» il criterio sia normativo che analitico-esplicativo dell'agire politico. Dove «comunicazione» è sinonimo di scambio di ragioni falsificabili ai fini di una intesa o Verständigung già iscritta nel procedimento linguisticocomunicativo.

I criteri della razionalità della comprensione linguistico-discorsiva diventano, per Habermas, criteri normativi esclusivi della razionalità politica. Di conseguenza consenso, solidarismo, civismo risultano benefici indotti dal dialogo o meglio dal «discorso» tra i cittadini, instaurato secondo criteri linguistico-comunicativi ideali. Insomma «l'intesa» sarebbe un dato intrinseco alla comunicazione pubblica, che viene insidiata dalle potenze anti-comunicative del denaro e del potere.

Ritengo che questa impostazione sia troppo semplicistica e svalorizzi le acquisizioni stesse della riflessione habermasiana: la centralità della dimensione comunicativa nella politica; la intimità tra Öffentlichkeit e linguaggio quotidiano; il nesso tra Öffentlichkeit e Zivilgesellschaft.

$\grave{E}$ ovvio che la comunicazione politica segua (debba seguire) le regole linguistiche; ma «l'intesa» come fine immanente del comunicare non può essere - a mio avviso - l'unico criterio per misurare la razionalità della politica. L'intrascendibilità del linguaggio, i criteri della sua comprensione/comunicazione (la trasparenza linguistica e la falsificabilità degli argomenti) sono cose assai diverse dai contenuti sostantivi su cui ci si intende (o non ci si intende) politicamente. Detto con un paradosso: in

${ }^{10}$ Cfr. tra l'altro, Habermas (1991) e Die zweite Lebenslüge der Bundesrepublik: Wir sind wieder «normal» geworden, in «Die Zeit», 51, 1992. Ho affrontato il dibattito sul «patriottismo costituzionale» in Rusconi (1993, cap. V). 
politica (ma non solo!) ci si può annientare reciprocamente proprio perché ci si è perfettamente capiti.

Tocchiamo qui uno dei limiti più seri dell'impostazione habermasiana - un'impostazione «filosofica» che ignora completamente la rilevanza delle diverse forme concrete del comunicare pubblico-politico. Nel concetto generico di «telos dell'intendersi» habermasiano trovano posto indistintamente il semplice consenso istituzionale alla democrazia, le più svariate forme del compromesso e patto sociale e i contenuti, anche più radicali, del solidarismo sociale. $\mathrm{Ma}$ - obietto - consenso istituzionale, patto sociale, solidarismo, pur appartenendo allo stesso orizzonte comunicativo, sono fenomeni assai diversi dal punto di vista logico e fattuale e quindi della loro performance comunicativa. Un conto, ad esempio, è l'adesione alle regole democratiche e costituzionali, il consenso competitivo a favore dell'una o dell'altra parte politica, un altro conto è la messa in atto di un solidarismo civico per fronteggiare difficoltà o emergenze particolari.

Credo che Habermas consideri irrilevanti queste ovvie constatazioni perché è inconsciamente prigioniero del criticismo francofortese che lo porta a vedere l'autenticità del «discorso pubblico» esclusivamente nella forma del dissenso democratico. E non sembra rendersi conto che lo stesso dissenso non è univoco né lineare nei suoi criteri e finalità. Non si può infatti mettere sullo stesso piano una manifestazione ambientalista e uno sciopero contro la disoccupazione, una marcia contro il razzismo e una dimostrazione per l'equità fiscale. Insistere sulla omologia «comunicativa» di queste manifestazioni (elevate addirittura a forme di kommunikative Macht) in contrapposizione alle ottuse incomunicanti «potenze sistemiche» - mi sembra un modo di ragionare molto fragile ${ }^{11}$ a fronte dei problemi sollevati.

1 Su questa strada invece continua a muoversi Jürgen Habermas anche nel suo ultimo lavoro (1992). In esso l'autore vede nello Stato di diritto «una risposta coerente alla questione di come possono essere istituzionalizzate le forme esigenti di comunicazione di una opinione e volontà politica democraticas (p. 361). Al concetto discorsivo di democrazia corrisponde l'immagine di una società decentrata che, attraverso la sfera politica pubblica, articola differenti arene per identificare e trattare in modo differenziato problemi sociali generali. Il sistema politico non è né il vertice né il centro della società, ma un sistema d'azione accanto ad altri. Questa visione «senza centro» del sociale coesiste con l'idea di una Lebenswelt come dato ultimo, invalicabile, insieme con la costituzione linguistica delle forme vitali socio-culturali (sprachliche Verfassung soziokulturellen Lebensformen). Partendo da questa invalicabilità del momento comunicativo Habermas rimprovera a molte teorie correnti della politica (teoria pluralista, teoria sistemica, teoria economica della democrazia) di avere un concetto «empiristico» di pote- 


\section{Razionalità comunicativa e agire politico}

La procedura tramite cui si raggiunge e si pratica di fatto la democrazia non solo rispecchia raramente la razionalità «discorsiva» à la Habermas, ma ne è spesso il sostituto. Infatti è soltanto nel confronto/scontro politico che si verifica quale intesa si instaura con l'avversario o il concorrente. Ciò che determina la qualità della comunicazione politica sono i contenuti dell'intesa, non la ricerca dell'intesa in quanto tale.

Nella comunicazione politica non si può escludere che l'altro non voglia accettare le nostre ragioni, pur approfittando della nostra disponibilità al dialogo. A questo punto è «razionale» il non farsi ingannare. Al limite: se il rapporto comunicativo con l'avversario mette in pericolo la nostra integrità, si può e si deve negare «l'intesa» con l'avversario (come accade nei casi estremi dei contatti con il terrorismo o con altre forme di estremismo e fondamentalismo ideologico-politico). In questi casi politica razionale consiste nel valutare e nell'assumersi la responsabilità (anche morale) della rottura o della impraticabilità del dialogo.

Insomma, se vogliamo guadagnare un criterio di razionalità nella comunicazione politica, che abbia rilevanza normativa, dobbiamo abbandonare il semplice paradigma dell'intendersi linguistico, per ricercarlo nella assunzione del rischio di tenere aperto un dialogo in assenza delle sue condizioni ottimali - addirittura nell'eventualità che esso venga negato o manipolato dalla controparte. La razionalità politica si presenta dunque come combinazione di accortezza «strategica» e disponibilità «comunicativa».

Chiarito questo passaggio, un altro modo per affrontare in positivo questa problematica può essere l'uso dei due paradigmi dell'argomentare e del negoziare (nel significato di Jon Elster). Il primo riguarda l'argomentazione razionale imparziale e il secondo la negoziazione di benefici virtualmente comuni, dai quali gli individui (o le parti) contraenti traggono maggiore o minore beneficio, a seconda della loro abilità nel contrattare

re, cieco verso la dimensione comunicativa. In realtà Habermas, preoccupato esclusivamente della virtualità solidaristica in ogni fenomeno socio-politico, non fa nessuno sforzo per vedere le componenti strumentali-strategiche che sono presenti in ogni azione politica, anche in quella radical-democratica da lui gratificata come espressione della razionalità comunicativa. 
o nel persuadere. Argomentare e negoziare appartengono all'universo del comunicare, ma investono risorse e strategie comunicative assai diverse ${ }^{12}$. Dialogare/argomentare e cooperare/ negoziare non sono omologhi o collocabili lungo un continuum logico che pretende di valere anche come misura etica. Se decidiamo di dialogare/cooperare, non lo facciamo in forza della intrinseca cogenza all'intendersi presuntivamente presente nel linguaggio e come tale trasmessa alla pratica cooperativa, ma perché nella comunicazione con i nostri interlocutori si crea una situazione di «fiducia» nel senso detto sopra. È una condizione contingente, precaria, sempre rinnegabile - ma è essa che qualifica in concreto la comunicazione e quindi l'agire politico.

Dunque: se è sterile una teoria politica che mette in contrasto comportamento strategico-utilitario e comportamento comunicativo, principio del vincere/perdere e principio dell'intendersi, paradigma del negoziare e paradigma dell'argomentare, logica dell'utilità e logica dell'identità, allora una teoria adeguata deve cogliere l'agire politico come un insieme di interazioni che combinano sistematicamente atti di intesa e atti di defezione, sostenuti da argomenti imparziali e da interessi negoziati.

Queste considerazioni valgono in generale per qualunque attore politico (cittadini individuali, raggruppamenti o partiti, rappresentanti di una istituzione). Ma hanno un rilievo del tutto particolare se sono riferite al «governo» inteso come attore politico che rappresenta ed esprime operativamente il complesso delle istituzioni politiche. Il governo infatti è l'agente istituzionalmente deputato a far «comunicare» e «cooperare» i cittadini nel senso detto sopra, con tutte le complicazioni che abbiamo visto.

Facciamo un altro passo in avanti. Questo saggio ha preso l'avvio dalle disfunzioni e dalle inefficienze che mettono a nudo carenze di orientamento e di autorevolezza del governo (italiano) con effetti di disgregazione identitaria al punto che non pochi cittadini sembrano mettere in dubbio il valore stesso dell'appartenenza alla comunità nazionale. $\mathrm{La}$ «secessione» virtuale o minacciata della nazione rappresenterebbe un caso particolarmente drastico di rottura «comunicativa» tra i cittadini membri di una comunità politica.

12 Jon Elster parla di criteri di validità opposti a criteri di credibilità. I primi dipendono dalla cogenza logica dell'argomento migliore, i secondi dalla forza della persuasione che utilizza tecniche comunicative più complesse e incontrollabili logicamente. Su questo si vedano i lavori citati nella n. 9 . 
Il quesito iniziale se e come le virtù civiche possano surrogare deficit della politica governativa si ripresenta qui in termini estremamente seri come interrogativo sulle capacità di un'intera classe politica di attivare risorse comunicative in grado di affrontare questa sfida.

Se si rompe il vincolo nazionale

In questa sede non entro nel merito della plausibilità o veridicità delle assicurazioni dei leader leghisti di non mirare alla secessione, ma solo al federalismo regionale in un quadro di unità formale della nazione. Il dibattito pubblicistico su questi temi non offre solidi elementi di valutazione ${ }^{13}$. Qui mi preme soltanto fare alcune congetture in linea con l'impianto analitico seguito sinora.

Ribadiamo che se in Italia dovessero verificarsi iniziative disgregatrici dell'unità nazionale, la causa prima va ricercata non nel revival di identità etno-culturali regionali più o meno inventate, ma nelle conseguenze del fallimento del sistema politico italiano. Alle sue insufficienze e inadempienze va imputato anche il fatto che le iniziative di riforma istituzionale regionale-federale, ora frettolosamente avanzate per contrapporsi al leghismo e salvaguardare il principio unitario, non sono sostenute da un serio ripensamento culturale dell'idea di nazione democratica. È piuttosto un appello ai buoni sentimenti e alla buona tradizione, scarsamente credibile sulla bocca di una classe politica in gran parte screditata. Di conseguenza, se il mutamento politico-istituzionale dovesse configurarsi di fatto come un cedimento contrattato con il leghismo, è difficile evitare che, dietro ad una qualche facciata di unità formale della nazione, si imbocchi la strada dei separatismi.

La prospettiva federalista leghista gode di tanta attrazione e tensione polemica contro la nazione storica perché alla sua base si è prodotta la fusione tra atteggiamenti utilitario-strumentali e atteggiamenti identitari che, in contesti storici diversi, è stata ti-

${ }^{13}$ Ho abbozzato un'analisi del leghismo, anche sulla scorta di recenti pubblicazioni - ad esempio, Diamanti (1993) - nella relazione L'identità nazionale e la sfida leghista, presentata al Convegno della Giunta centrale per gli studi storici «Nazione e nazionalità in Italia dalla prima guerra mondiale ai nostri giorni», Trieste 15-18 settembre 1993, di prossima pubblicazione negli Atti del Convegno stesso. 
pica della costruzione delle nazioni storiche e della loro forza di attrazione. Oggi l'intensa identificazione territoriale ${ }^{14}$, il linguaggio riconoscibile ${ }^{15}$, la rivendicazione del controllo diretto delle proprie risorse ${ }^{16}$ servono a de-costruire la nazione storica a vantaggio di entità regionali, che funzionalmente e strutturalmente assomigliano a micronazioni.

Più esattamente in Italia si profila una variante di un fenomeno già registrato in altre aree del mondo: la etnodemocrazia. $\mathrm{Nel}$ nostro caso etnodemocratica è la pratica dei diritti politici e civili esercitata entro confini autodefiniti e autodefinibili (ed eventualmente coercitivamente controllabili), ritagliati entro e contro una precedente configurazione geografica e politica nazionale appunto. L'etnodemocrazia così intesa fa sentire pienamente cittadino soltanto chi si trova su un territorio dichiarato «proprio» in antagonismo con il territorio-nazione, disinteressandosi delle conseguenze generali. Principio universalistico (democrazia) e principio particolaristico (etnolocalismo) trovano la loro paradossale sintesi.

Non credo che il leghista, anche più estremista, che sogna la sua «Repubblica del nord» pensi a restrizioni di diritti civili e politici per chi dovesse venire dalle altre due «repubbliche». $\mathrm{Ma}$ un effetto perverso di alcuni atteggiamenti estremisti sarebbe un diverso valore dei contenuti sociali della cittadinanza nella macroregione settentrionale rispetto a quella meridionale. Quest'ultima, nella sua nuova «autonomia», dovrà rinegoziare da ovvie posizioni di debolezza un'infinità di regole che porteranno ad una profonda alterazione dell'attuale pacchetto di diritti sociali degli abitanti delle regioni meridionali ${ }^{17}$. Per il mo-

14 È importante non sottovalutare questo aspetto - considerato a torto un po' folkloristico. La Lega è riuscita ad attivare aree culturalmente mortificate dall'ideologismo tradizionale offrendo (magari inventando) una forte identità, radicata nella propria terra, nella propria storia, nella propria cultura - arrivando talvolta a parlare di etnia e nazione a dimensione regionale.

15 La Lega, dopo iniziali incertezze circa un ipotetico ripristino dei dialetti locali, ha coniato un suo linguaggio peculiare, immediatamente riconoscibile dalla gente leghista. È un linguaggio «popolano» - come ama dire Umberto Bossi. La sostanza di questo linguaggio non sta tanto in frammenti dialettali, deliberatamente sussunti nella lingua parlata nazionale, quanto nella esibita grevità $\mathrm{e}$ aggressività delle sue sortite in aperta contrapposizione al linguaggio gergale del «politichese» romano, percepito dalla gente come vagamente truffaldino.

16 In questa ottica rivendicazioni significative, ma di portata limitata, attinenti la pressione fiscale o il diritto della (propria) regione all'imposizione e controllo fiscale acquistano un valore simbolico difficilmente sottovalutabile.

${ }^{17}$ Non c'è dubbio che tutto questo risponde alle intenzioni di quei leghisti che in 
mento, è difficile distinguere nel programma e nella strategia del leghismo la funzionalità di un progetto regionale-federale dal semplice disimpegno dai legami storici che motivano alla solidarietà nazionale. Un fatto è certo: la minacciata ritrattazione dell'appartenenza nazionale non è più soltanto una rescissione di legami culturali e identitari, ma implica una revisione dei criteri e dei contenuti concreti di una cittadinanza che sino ad oggi ha conservato, bene o male, caratteri universalistici nella sua forma nazionale. Allo stato attuale, nel progetto leghista è latente un potenziale etnocentrico che entra in collisione con quella concezione universalistica della cittadinanza, che attraverso vie differenti ${ }^{18}$ ha trovato il suo veicolo nello stato-nazione democratico.

D'altra parte è innegabile che $\mathrm{i}$ ceti e i gruppi sociali che oggi riscoprono il valore della nazione unitaria, con il mantenimento della sua forma attuale, schierandosi magari a fianco del nuovo movimento di destra, attuano una strategia di difesa particolaristica. È irrilevante qui decidere se si tratta di ceti effettivamente deboli o semplicemente clientelari che temono di essere penalizzati da qualunque innovazione dello status quo. Ciò che conta è che essi reagiscono non secondo i principi di una cittadinanza universalistica - nel senso detto sopra - ma secondo criteri particolaristici. In altre parole, sia il leghismo nordista che la reazione neo-nazionalista centro-meridionale, lasciati a se stessi, minano i fondamenti della nazione democratica.

\section{La «nazione dei cittadini»}

Riepiloghiamo. Le istituzioni politiche democratiche sono vitali quando nel loro funzionamento realizzano le due logiche strumentale e identitaria in modo soddisfacente per $i$ loro fruitori/cittadini. Non sorprende allora che la disfunzionalità o l'inefficienza delle istituzioni incidano anche sulla loro pretesa di riconoscimento identitario da parte dei cittadini stessi. Il caso italiano è esemplare nel mostrare come inefficienza istitu-

un sol colpo vogliono punire la cattiva politica meridionalista condotta sinora dallo stato centrale, smobilitare l'elefantiaco e improduttivo stato assistenziale, insediato soprattutto al Sud, e, per soprappiù, liberarsi della mafia che ai loro occhi è lo stigma del Mezzogiorno come tale.

${ }^{18} \mathrm{Cfr}$. l'ampia ricerca storico-politologica di Giovanna Zincone (1992). 
zionale e disidentificazione siano strettamente collegate, disincentivando il solidarismo civico al punto da mettere in discussione virtualmente lo stesso principio nazionale.

Reciprocamente la virtù civica ha un doppio carattere: è l'aspettativa (utilitaria) di un vantaggio comune o di bene pubblico ottenuto tramite cooperazione, anche nel caso fosse differito nel tempo; ma insieme è la disponibilità ad accollarsi anticipatoriamente (sulla fiducia) un investimento di costi supplettivi, in nome di una identificazione solidale, che ha $\mathrm{i}$ suoi referenti nella nazione storica (che può coesistere con differenti formeStato).

Se in questo quadro d'analisi collochiamo il tema della cittadinanza, concepita come la titolarità di accesso a determinati beni pubblici che hanno forma di diritti (civili, politici, sociali) ma che sono ottenibili soltanto grazie a costi equamente divisi, allora la cittadinanza appare non semplicemente come un catalogo di diritti soggettivi, ma come un vincolo di reciprocità tra $\mathrm{i}$ membri di una stessa comunità politica. Essa implica non soltanto uguaglianza delle domande e delle prestazioni ma anche obblighi di solidarietà, soprattutto in condizioni di oggettiva disparità di risorse di vita tra i membri di una comunità politica. In altre parole, in determinate circostanze il vincolo della cittadinanza spinge (dovrebbe spingere) alcuni cittadini a contribuire o a «cooperare più degli altri» ${ }^{19}$. Come abbiamo avvertito sin dall'inizio, questo tipo di solidarismo non è «l'altruismo» disinteressato, motivato da convinzioni morali e/o religiose soggettive, ma è un comportamento intrinseco alla condizione della cittadinanza come tale.

$\mathrm{Ma}$ in nome di che cosa un governo democratico può chiedere questo investimento fiduciario? Quali argomenti può usare, soprattutto se con esso intende compensare deficienze di gestione o addirittura di sistema? I motivi che possono convincere i cittadini al rischio di essere solidali, ad investire fiducia nel senso detto sopra, non possono che basarsi sul riconoscimento che momenti importanti della loro propria storia, cultura e identità individuale sono parte dell'identità collettiva stessa della nazione d'appartenenza. È questo riconoscimento identitario

${ }_{19}$ Per lo sviluppo analitico di questa affermazione rimando di nuovo al saggio di Michael Taylor citato nella nota $\mathrm{n}$. $7 \mathrm{e}$, per un approccio più sistematico, al suo volume (1987). 
che si traduce in accettazione di vincoli solidali, non a titolo privato ma a titolo pubblico, perché toccano lo status di cittadini costituiti in nazione ${ }^{20}$.

Una nazione dei cittadini, così concepita, ha un rapporto diretto con la «società civile», intesa come luogo in cui vengono criticamente riconfermati quei legami di storia e cultura comune che favoriscono il comportamento cooperativo. Naturalmente la «società civile» continua ad essere anche la sede del confronto/scontro degli interessi materiali legittimi e del calcolo dei vantaggi e dei benefici privati. Ma questo conferma semplicemente la doppia razionalità di tutto ciò che attiene all'ambito «civico/civile».

Nel corso delle nostre riflessioni, al lettore non sarà sfuggita una sorta di oscillazione nel definire o meglio nel giustificare l'esercizio della virtù civica. Questa infatti per un verso appare come la culminazione e la sanzione della razionalità complessiva di un sistema politico funzionante. Per un altro verso invece è una risorsa di tipo storico-identitario, strategicamente richiesta (dal governo democratico) per sostenere un sistema politico o semplicemente una politica in difficoltà. In effetti, il paragrafo dedicato a «Le ragioni del civismo», presenta queste ultime

20 Queste affermazioni sembrano convergere con certe tesi comunitariste di Charles Taylor ad esempio, che parla delle «repubbliche» che funzionano «nel senso che parte di ciò che lega insieme le persone è la storia comune» (1992). Da questa condizione nasce il patriottismo inteso come «identificazione con gli altri in una impresa comune particolare», o ancora come «un'identificazione comune con una comunità storica fondata su certi valori». Collocandosi a metà strada tra l'appartenenza familiare/amicale e l'altruismo universalistico, questo patriottismo «repubblicano» consentirebbe il concreto funzionamento della comunità politica, che sfuggirebbe invece alle spiegazioni del liberalismo «atomistico» e procedurale che mantiene una concezione strumentale e opportunistica del bene comune. Nella sua critica Taylor usa molti argomenti plausibili, ma non convince nella contrapposizione filosofica dei due modelli repubblicano e liberale (facendone, tra l'altro, una questione «ontologica» della comunità e dell'identità). In ogni caso il suo modo di procedere va nella direzione opposta a quella da noi intrapresa. La «nazione dei cittadini», di cui parliamo, non è né il comunitarismo repubblicano di Taylor né l'astratto proceduralismo dei diritti e degli interessi da lui imputato al modello liberale. Va aggiunto che molte osservazioni di Taylor sono intimamente legate alla specifica esperienza e cultura politica americana, la cui generalizzazione e trasposizione in altri contesti politico-culturali richiederebbe un lungo lavoro di mediazione interpretativa. A questo proposito, $\mathrm{mi}$ si consenta soltanto una battuta. Per Taylor uno degli esempi concreti a favore del patriottismo repubblicano è la «capacità di sdegno» emersa negli USA contro Nixon nel caso Watergate. Ovvero soltanto «l'identificazione patriottica», nessun altro atteggiamento liberale-strumentale-atomistico, avrebbe potuto suscitare tanta emozione pubblica. Se trasportassimo questa affermazione al caso italiano, potremmo dire che lo sdegno popolare per Tangentopoli nasce da una «identificazione patriottica»? 
come un intreccio variante di incentivi utilitari, di argomenti universalistici e di motivazioni storico-identitarie; il presente paragrafo invece insiste sulle motivazioni storico-identitarie, quasi che soltanto ad esse - in ultima istanza - faccia assegnamento la virtù civica.

In realtà, più che di una oscillazione, si tratta di una variazione per estensione, più o meno ampia e inclusiva, delle ragioni del civismo cui la comunicazione politica può di volta in volta far ricorso. L'adozione dell'una o dell'altra motivazione dipende dagli uomini di governo, oltre che dalle circostanze e dalla natura delle sfide cui un sistema democratico è esposto.

In questa operazione acquista il suo pieno significato il concetto di strategia comunicativa del ceto dirigente di un paese democratico. Tramite essa viene in primo piano la statura morale e culturale, la «competenza comunicativa» degli uomini di governo e dei loro argomenti che fanno appello al solidarismo nazionale.

A ben vedere, è un'intera cultura politica che è chiamata alla prova della sua credibilità.

\section{Riferimenti bibliografici}

Arrow, K.J. (1987), Equilibrio, incertezza e scelta sociale, Bologna, Il Mulino.

Austin, J.L. (1962), How to Do with Words, Cambridge, Harvard University Press; trad. it. Quando dire è fare, Torino, Marietti, 1965.

Diamanti, I. (1993), La Lega. Geografia, storia e sociologia di un nuovo soggetto politico, Roma, Donzelli.

Elster, J. (1989), The Cement of Society: A Study of Social Order, Cambridge, Cambridge University Press; trad. it. in corso, Bologna, Il Mulino.

- (1993a), Argomentare e negoziare, Milano, Anabasi.

- (1993b), Come si studia la società, Bologna, Il Mulino.

Ferrara, A. (a cura di) (1992), Comunitarismo e liberalismo, Roma, Editori Riuniti.

Gambetta, D. (a cura di) (1989), Le strategie della fiducia, Torino, Einaudi.

Habermas, J. (1991), Cittadinanza e identità nazionale, in «Micromega», n. 5, pp. 123-146.

- (1992), Faktizität und Geltung. Beiträge zur Diskurstheorie des Rechts und des demokratischen Rechtsstaats, Frankfurt a.M., Suhrkamp. 
Lewin, L. e E. Vedung (a cura di) (1980), Politics as Rational Action, Dordrecht, Reidel.

MacIntyre, A. (1992), Il patriottismo è una virtù?, in Ferrara (1992), pp. 55-76.

Midgaard, K. (1980), On the Significance of Language and Richer Concept of Rationality, in Lewin e Vedung (1980), pp. 83-97.

Riker, W. (1980), Political Trust as Rational Choice, in Lewin e Vedung (1980), pp. 1-24.

Rusconi, G.E. (a cura di) (1989), Giochi e paradossi in politica, Torino, Einaudi.

- (1993), Se cessiamo di essere una nazione, Bologna, Il Mulino.

Sartori, G. (1990), Elementi di teoria politica, Bologna, Il Mulino.

Taylor, C. (1992), Il dibattito tra sordi di liberali e comunitaristi, in Ferrara (1992), pp. 137-168.

Taylor, M. (1987), The Possibility of Cooperation, Cambridge, Cambridge University Press.

Taylor, M. e H. Ward (1989), La fornitura di beni pubblici: un'applicazione della teoria dei giochi, in Rusconi (1989), pp. 73-103.

Zincone, G. (1992), Da sudditi a cittadini, Bologna, Il Mulino. 